Original Article

\title{
OPTIMIZATION AND CHARACTERIZATION OF RIVASTIGMINE-LOADED NANOSTRUCTURED LIPID CARRIERS
}

\author{
SWATHI GANNA1 ${ }^{1}$, SAI MANOGNA KOTAKADI ${ }^{1}$, RESHMA ANJUM MOHAMMED ${ }^{1}$, MANNUR ISMAIL SHAIK², \\ JOHN SUSHMA NANNEPAGA ${ }^{*}$
}

${ }^{1}$ Department of Biotechnology, Sri Padmavati Mahila Visvavidyalayam, Tirupati 517502, India, ${ }^{2}$ Faculty of Fisheries and Food Science, University of Malaysia Terengganu, KulaNerus 21030, Malaysia

Email: johnsushma@gmail.com

Received: 28 Jan 2021, Revised and Accepted: 22 Mar 2021

\section{ABSTRACT}

Objective: The objective of the present study was to develop Nanostructured lipid carriers (NLCs) for improvement in the oral bioavailability of RT.

Methods: RT-loaded NLCs were prepared by high shear homogenization technique using fish oil and flaxseed oil respectively. The prepared RTNLCs were characterized using a phase-contrast microscope, scanning electron microscope (SEM), atomic force microscope (AFM), Fourier transform-infrared spectroscopy (FTIR) and differential scanning calorimetry (DSC). Further, particle size, entrapment efficiency and sustained release of the drug were also studied.

Results: SEM results revealed that the RT-NLCs were spherical in shape with a smooth surface. AFM results confirmed the formation of spherical particle dispersions by the NLCs in nanoscale. FTIR spectroscopy and DSC analyses revealed that there is no chemical interaction between the ingredients of RT-NLCs. The particle size of the RT-NLCs was found to be exponentially decreased with the increase in a surfactant solution.

Conclusion: The results confirmed pronounced improvement in entrapment efficiency of optimized formulation of RT-NLCs. In vitro, drug release studies showed that RT-NLCs were capable of releasing the drug in a sustained manner. The experimental results showed that the NLCs are potential carriers for providing sustained delivery of rivastigmine.

Keywords: NLCs, Rivastigmine, SEM, AFM, DSC, FTIR

(C) 2021 The Authors. Published by Innovare Academic Sciences Pvt Ltd. This is an open access article under the CC BY license (https://creativecommons.org/licenses/by/4.0/) DOI: https://dx.doi.org/10.22159/ijcpr.2021v13i3.42093 Journal homepage: https://innovareacademics.in/journals/index.php/ijcpr

\section{INTRODUCTION}

Alzheimer's disease (AD) is a progressive disorder that causes brain cells to degenerate leading to dementia, cognitive and personality inabilities. Hence, it is considered as a consequential obstruction not only for patients but also for their relatives and the whole society [1]. There are diverse etiological factors for $A D$ such as genetics, environmental factors and general lifestyles [2]. It is specified pathologically with the aggregation of amyloid and tau protein in the brain regions [3]. In the development of therapeutics for CNS diseases, one of the greatest challenges is the formulation of a drug with adequate penetration through the blood-brain barrier (BBB) [4]. As most of the drugs fail to cross the BBB, targeting drugs to the brain is an important issue for pharmaceutical research. Drug development for $\mathrm{AD}$ is not much noticeable so far as $98 \%$ of smallmolecule drugs and $100 \%$ of all large molecule drugs are unable to cross the BBB [5].

Rivastigmine (RT), a novel acetylcholinesterase inhibitor, is used for the treatment of $\mathrm{AD}[6]$. But to overcome the short half-life $(1.5 \mathrm{~h})$ of RT [7], a sustained-release formulation with reduced side effects and simplified dosage regimens are suggested to improve treatment adherence and compliance [8]. Many formulations were prepared for the sustained release of RT such as encapsulation in liposomes [9], emulsions and nanoparticles [10-12]. Nanoparticles are identified as one of the most favourable and versatile drug delivery systems to provide therapeutic agents while efficiently delivering them into the damaged areas of the brain [13]. Nanostructured Lipid carriers are the second-generation lipid carriers developed to overcome problems associated with solid lipid nanoparticles and are utilized in various therapeutic approaches. NLCs are considered as a standard drug carrier system due to their characteristic ability to cross the BBB [14]. When compared to other polymeric materials lipid molecules are more beneficial due to their physiological and biodegradable nature, which will reduce the adverse effects and persistent toxicity. NLC matrix consists of a mixture of different lipid molecules that leads to deformity in the matrix to accommodate more drug molecules [15]. NLCs have several advantages such as increased solubility, permeability, bioavailability and also the capacity to improve storage stability, high loading capacity, prevention of the drug expulsion during storage, reduced adverse effects and tissue-targeted drug delivery over conventional carriers $[16,17]$. New advances in the field of Alzheimer's disease research is drug delivery to the brain through NLCs. The novelty of the work lies in the successful preparation of NLCs with fish oil and flaxseed oil and loading of RT into the NLCs and their characterization studies.

\section{MATERIALS AND METHODS}

\section{Materials}

Rivastigmine tartrate (RT) is commercially available as Rivamer. Fish oil and flaxseed oil was obtained from the local market, Tirupati, Andhra Pradesh, Tween 80 and other chemicals were obtained from Hi media and Merck chemical companies.

\section{Preparation of RT loaded NLCs}

In the present study, RT-NLCs were prepared using variable lipid $(10 \%, 20 \%, 30 \%)$ and $\mathrm{RT}(0.5,1,1.5 \mathrm{mg})$ ratios by melting emulsification coupled with high shear homogenization technique. The procedure was carried out in 9 different batches with variable drug/lipid ratios as shown in Table.1. RT-NLCs-1,2,3 contains $0.5 \mathrm{mg}$ of RT, RT-NLCs-4,5,6 contains 1.0 mg of RT, RT-NLCs-7,8,9 contains $1.5 \mathrm{mg}$ of RT with variable lipid concentrations of $10 \%, 20 \%, 30 \%$ to identify the suitable lipid ratios for better entrapment efficiency of the drug and also for sustained release of the RT present in the formulations. Briefly, in a melted lipid phase composed of (fish and flaxseed oil) an appropriate amount of RT (table 1) was added. After adding RT, the lipid mixture was kept for $5 \mathrm{~min}$ and then mixed with $1 \%$ hot tween 80 aqueous surfactant. The hot emulsion resulted by 
mixing lipid and aqueous phases at $85{ }^{\circ} \mathrm{C}$ and it was subjected to external mechanical energy by high shear homogenization at 25,000 rpm for a period of $10 \mathrm{~min}$ (using an Ultra Turrax® T18 Basic (IKA®, Germany). The resultant pre-emulsion was allowed to cool down at room temperature, with the formation of aqueous dispersions of RT-loaded NLCs. The composition of each RT-NLCs and some physicochemical characteristics are presented in table 1. Finally, the dispersions of RT-NLCs were lyophilized (-55 0C, $72 \mathrm{~h}$ ), by using a ScanVac-COOLSAFE-Freeze Dryer System equipment (Braunschweig, Germany)17, in order to remove the excess of water and to obtain a powder of RT-NLCs.

RT-NLCs formulations have been prepared by using fish and flaxseed oil $(10 \%, 20 \%, 30 \%)$ and variable RT concentration $(0.5$, $1.0,1.5 \mathrm{mg}$ ) as shown in table 1, with surfactant Tween 80.

\section{Characterization of RT-NLCs}

\section{Morphological and physicochemical characterization studies}

\section{Microscopic view of RT-NLCs}

A thin layer of the diluted formulations of RT-NLCs was observed by phase-contrast microscope (Olympus No. CX41RF) [18] and photomicrographs were taken using a digital camera.

\section{Scanning electron microscopy (SEM)}

The morphological characteristics of RT-NLCs were investigated using a scanning electron microscope (JEOL JSM-6700F) [19]. Thin films of the sample were prepared on a carbon-coated copper grid by just dropping a small amount of the sample on the grid. The extra solution was removed using a blotting paper and then the films on the SEM grid were allowed to dry.

\section{Atomic force microscopy (AFM)}

The morphology of the RT-NLCs was analyzed by an atomic force microscope (AFM-SolverNext, NT-MDT, Russia) [20]. A drop of RTNLCs suspension was placed on a thin aluminum sheet and dried at room temperature, prior to analysis of the sample in AFM.

\section{Fourier transform infrared (FTIR) spectroscopy}

The FTIR spectra of the RT-NLCs samples were recorded on the JASCO FT-IR5300 model spectrophotometer (Japan) [21] in the spectral range between 4000 and $400 \mathrm{~cm}^{-1}$ to identify functional groups in the RT-NLCs formulations.

\section{Differential scanning calorimetry (DSC)}

DSC is a useful tool to monitor the thermal behavior of materials and used to derive qualitative information about the physicochemical status of the drug in NLCs [22]. Each sample was sealed in an aluminum disc and purged with air at a flow rate of $40 \mathrm{ml} / \mathrm{min}$ and maintained the temperature at $50{ }^{\circ} \mathrm{C}-200{ }^{\circ} \mathrm{C}$ using Mettler STAR SW-
12 equipment. The DSC spectrum of the pure rivastigmine was compared with a mixture of the RT-NLCs.

\section{Measurement of zeta potential}

The particle size of RT-NLCs was measured by a particle size analyzer (Horiba scientific nanoparticle SZ100 Z). For the measurement, $100 \mu \mathrm{L}$ of the formulation was diluted with an appropriate volume of phosphate-buffered saline (PBS) at $\mathrm{pH} 7.4$ and the diameter was determined. Zeta potential of the RT-NLCs was determined by using Malvern Zetasizer Nano (Malvern Instruments Ltd., UK) [23]. $1 \mathrm{ml}$ of the sample was diluted to $10 \mathrm{ml}$ with distilled water, $5 \mathrm{ml}$ of this diluted sample was transferred to a cuvette and the zeta potential was measured. The measurement of zeta potential was based on the direction and velocity of particles under the influence of the known electric field.

\section{Drug entrapment efficiency}

The entrapment efficiency is also known as association efficiency. The RT-loaded NLCs are centrifuged at a high speed of 3500-4000 rpm for $30 \mathrm{~min}$ and the supernatant is assayed for non-bound drug concentration by UV-spectrophotometer. Entrapment efficiency was calculated by the following equation $[24,25]$.

$$
\% \text { Entrapment Efficiency }=\frac{\mathrm{RT}_{\text {Total }}-\mathrm{RT}_{\text {Supernatant }}}{\mathrm{RT}_{\mathrm{Total}}} \times 100
$$

Where RT total is the weight of the total incorporated drug and RT supernatant is the free drug analyzed in the supernatant layer.

Loading capacity of the drug was calculated as follows [24, 26].

$$
\% \text { Drug loading }=\frac{\text { TheamountofentrappeddruginNLCs }}{\text { Thetotalamountofdrug }} \times 100
$$

\section{RESULTS AND DISCUSSION}

\section{Preparation of the RT loaded NLCs}

In the present study, RT-NLCs were prepared using variable lipid $(10 \%, 20 \%, 30 \%)$, rivastigmine $(0.5 \mathrm{mg}, 1.0 \mathrm{mg}, 1.5 \mathrm{mg})$ ratios by using melting emulsification coupled with high shear homogenization technique. $1 \%$ Tween 80 was used as a surfactant which yields better stabilization of the dispersion system. The formation of RT-loaded NLCs was primarily confirmed by phasecontrast microscope (fig. 1), RT showed high affinity towards the organic phase because it was dissolved in the organic solvent, acetone. The different concentrations of RT were added drop-wise to the variable concentrations of the NLCs will enhance the drug entrapment efficiency which may lead to the uptake of RT by the brain microvessel endothelial cells.

The liquid dispersions were lyophilized at $-55^{\circ} \mathrm{C}$ for $72 \mathrm{~h}$ resulting in the powder forms of RT loaded NLCs. This powder was preserved at$4{ }^{\circ} \mathrm{C}$ and used for further characterizations.
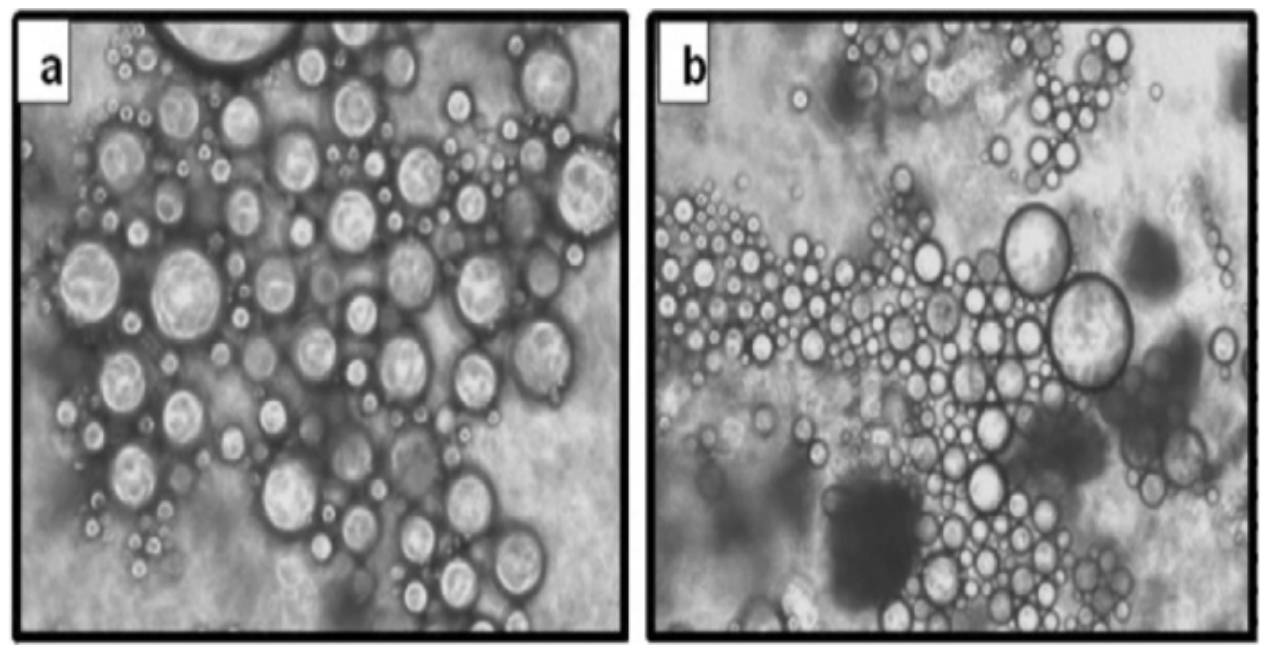

Fig. 1: Surface morphology study using phase contrast microscope in (a) NLCs1 and (b) NLCs9 


\section{Scanning electron microscopy studies of NLCs}

SEM study showed the discrete RT-NLCs with a rod, spherical shape particles (fig. 2). The spherical shape of RT-NLCs has been reported in previous findings [27]. The size distribution of RTNLCs indicated that they were more or less spherical in shape with an average diameter of $2 \pm 10 \mathrm{~mm}$, distributed in the range of $2-8$ $\mathrm{mm}$.
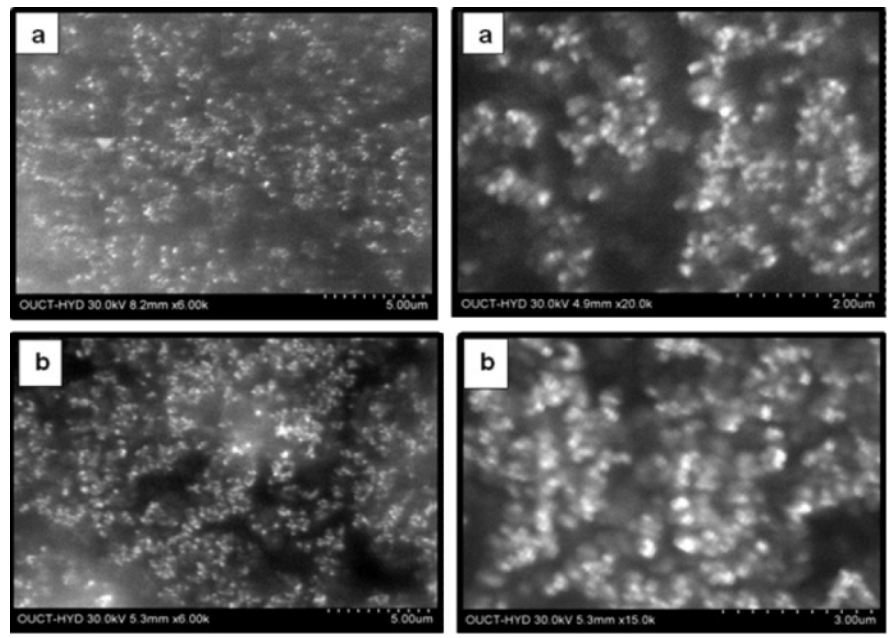

Fig. 2: Particle size distribution study using SEM in (a) NLCs1 and (b) NLCs9

\section{Atomic force microscopy (AFM)}

The morphology of the NLCs was analyzed by AFM as shown in fig. 3 . The images of formulations RT-NLCs1and RT-NLCs9 show particles with spherical morphology (fig. 3). In the photomicrographs, the particle diameters were approximately minimum-5.868 and maximum $10.27 \mathrm{~nm}$.

Fourier transform infrared (FTIR) spectroscopic analysis of NLCs

Fourier transform infrared spectroscopic analysis confirmed the chemical interaction between RT and NLCs. The FTIR spectra of RTNLCs at room temperature is shown in (fig. 4), the characteristic peaks of functional groups were observed at 2928, 1651, 1430, 1259, $1021 \mathrm{~cm}-1$ due to C-H stretch, C-C stretch, C-N stretch which indicates the presence of alkanes, aromatics, and aliphatic amines; there was a sharp peak at $1744.5 \mathrm{~cm}-1$ indicates the presence of esters, peak at $3379 \mathrm{~cm}-1$ was due to $0-\mathrm{H}$ stretching, indicates the presence of alcohols and phenols.

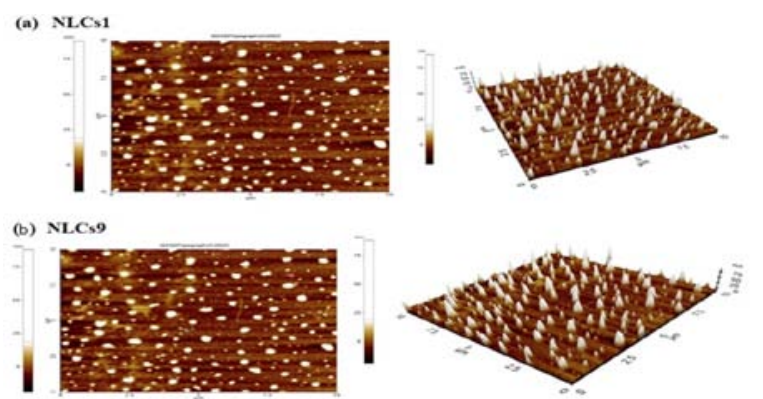

Fig. 3: Atomic force microscopic images of NLCs1 and NLCs9 2D and $3 D$ view

\section{Differential scanning calorimetry of RT-NLCs}

The differential scanning calorimetry was performed to investigate the melting behavior and crystalline structure of the lipid molecules and drugs in the nanoparticle preparations [28]. The allure of DSC curves reveals the endothermic peak of reference RT at $146.8{ }^{\circ} \mathrm{C}$, NLCs showed the endothermic peaks at $110.5^{\circ} \mathrm{C}$ (specific for fish oil), and another endothermic peak at $160.24{ }^{\circ} \mathrm{C}$ (specific for flaxseed oil). The endothermic peak of RT was completely absent at $146.8{ }^{\circ} \mathrm{C}$ of RT-loaded NLCs (fig. 5), which indicates the complete solubilization or conversion of RT from crystalline to amorphous form in the matrix of NLCs. Graph C showed a peak at $153.94{ }^{\circ} \mathrm{C}$, this indicates RT was not in the crystalline state in the formulation. This is in consonance with the previous reports [29]. The shifting of the endothermic peak indicates the interaction of the RT with the NLCs. The obtained results suggested that the RT-NLCs consisted of a homogeneous amorphous drug-polymer matrix (fig. 5).
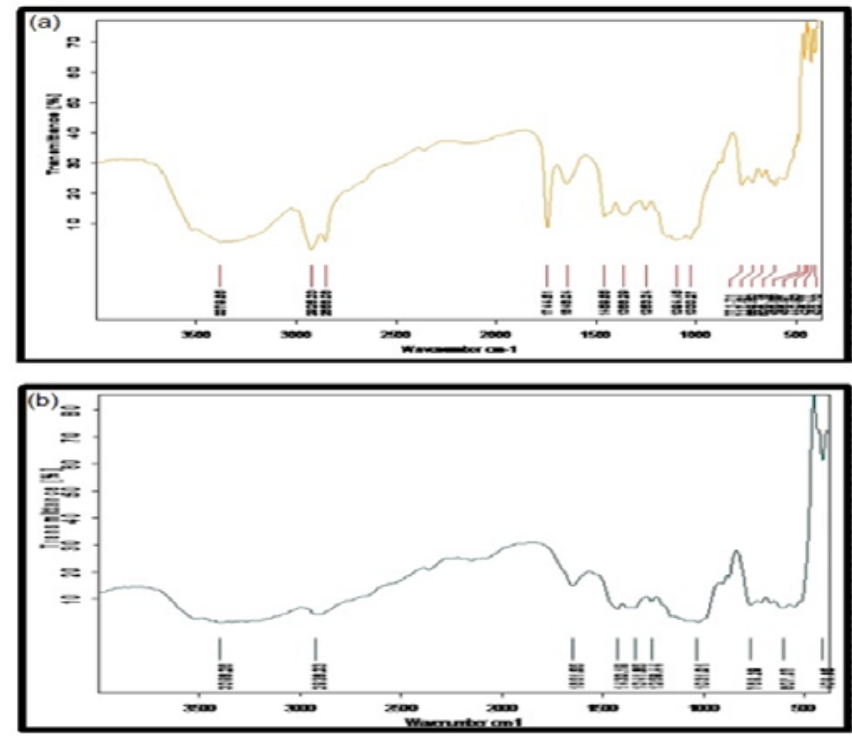

Fig. 4: FTIR spectra of (a) RT loaded NLCs and (b) RT

\section{Drug entrapment efficiency}

The determination of the entrapment efficiency (EE\%) is an essential stage in order to predict the suitability of NLCs based on omega-3 fatty acids, as efficient reservoirs for the RT. The entrapment of RT inside the lipid core was strongly associated with the RT and the flaxseed oil, fish oil concentrations. The EE\% was 
improved as the fish oil content has been increased to a maximum value of $30 \%$ (e. g. from $82 \%$ for RT-NLCs-1 prepared with $10 \%$ fish oil to $94.6 \%$ for RT-NLCs-9 prepared with $30 \%$ fish oil) (fig. 6) These results could be justified by the high solubility of RT into the NLCs which contain the liquid fish oil reservoir. In other words, a less ordered arrangement of the NLCs is accompanied by high entrapment efficiency. The EE of RT-NLCs-3,6,9 of $30 \%$ fish oil showed more RT entrapment than RT-NLCs of 1,4,7 prepared by $10 \%$ fish oil. Such type of EE in NLCs was reported in earlier studies.
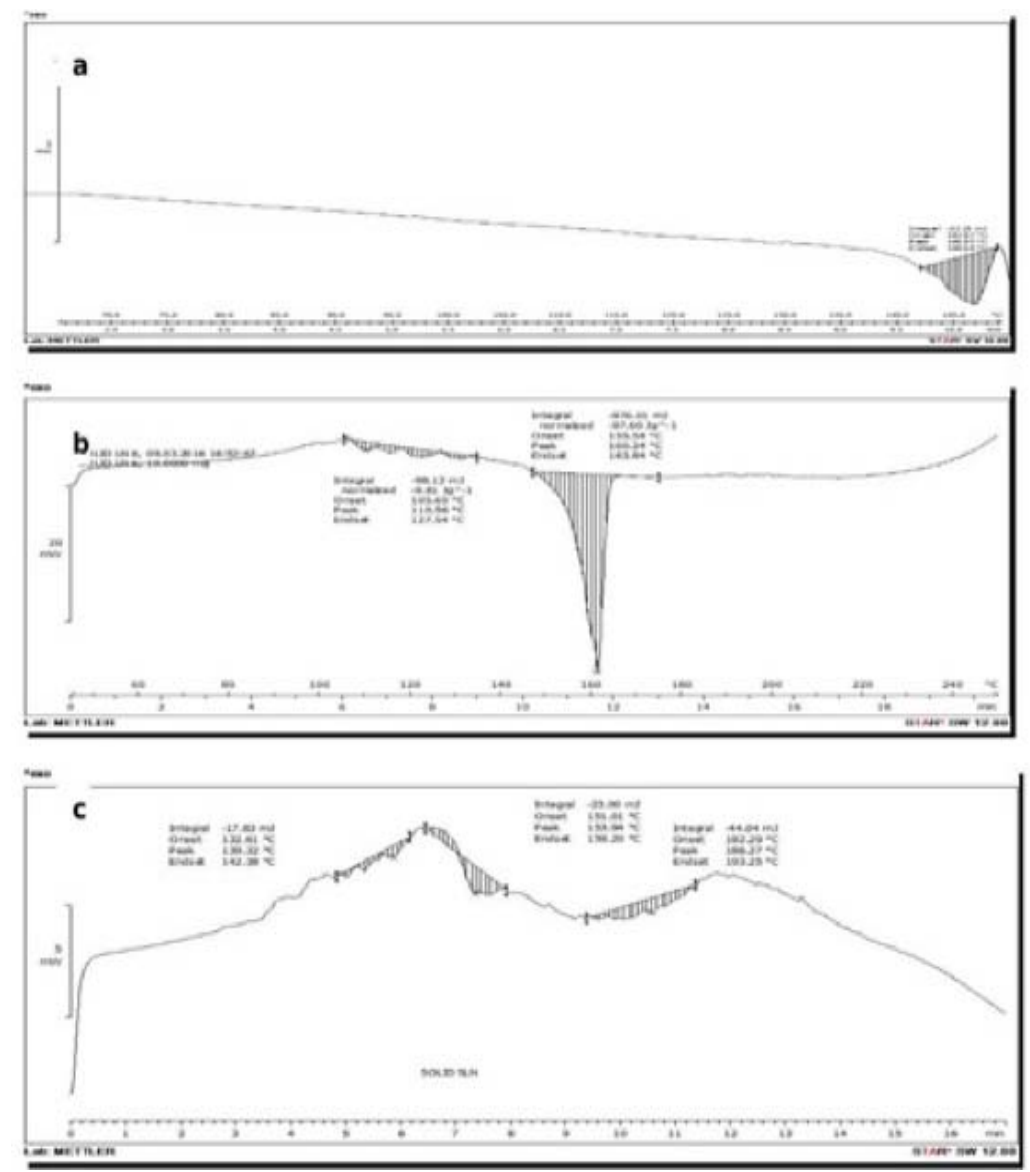

Fig. 5: DSC thermograms exhibiting the peak transition onset temperature of (a) RT (b) RT-NLCs1 (c) RT-NLCs9

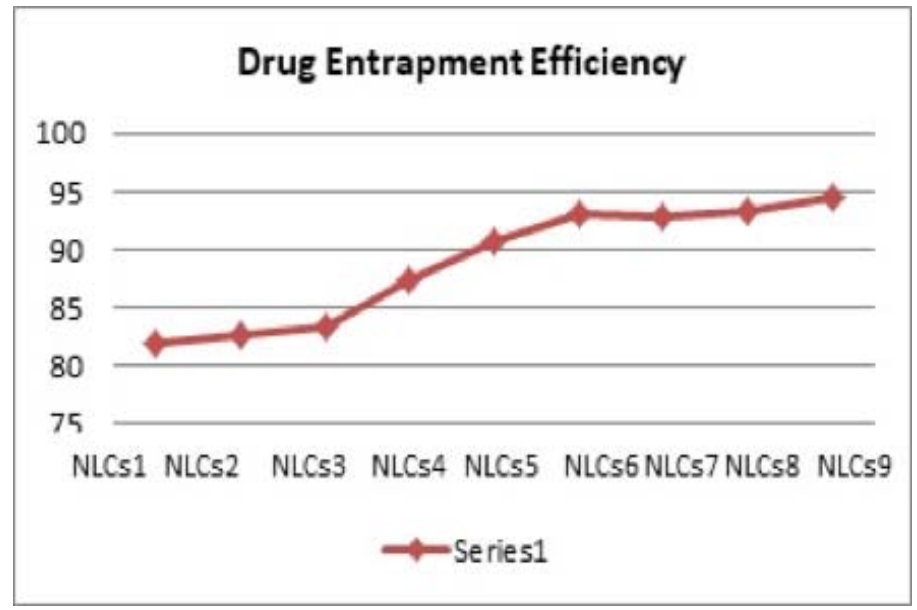

Fig. 6: Drug entrapment efficiency of RT-NLCs

\section{Particle size and zeta potential}

The particle size of RT-NLCs (1 and 9) showed a considerably smaller size with an average diameter of 1 to $100 \mathrm{~nm}$ of RT-NLCs (fig. 7). From the results, it was found that the zeta potential values of the RT-NLCs1 was-21.1 $\mathrm{mV}$ and NLCs9 was-16.4 mV (fig. 7) which imparts good stability of the RT-NLCs dispersion. Zeta potential is a significant tool in predicting the stability of NLCs during storage. Zeta potential values of more than $+30 \mathrm{mV}$ or less than $-30 \mathrm{mV}$ are considered enough for the stabilization of NLCs [30, 31]. 

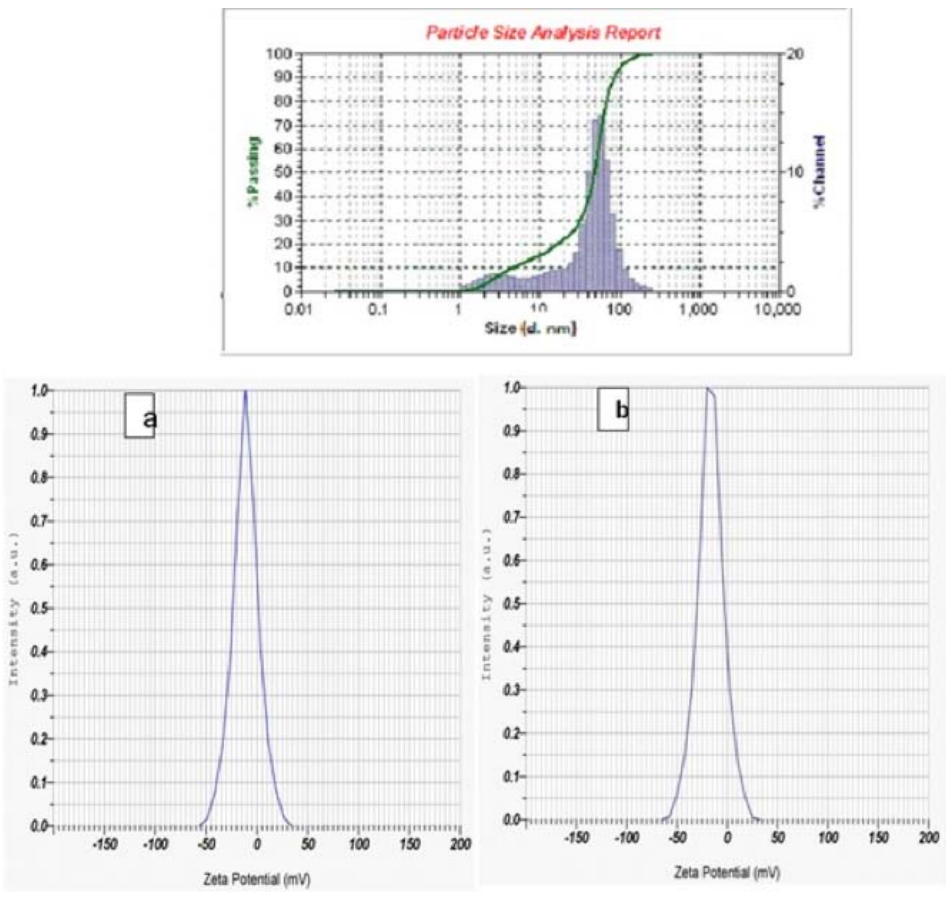

Fig. 7: Particle size analysis (upper image) and zeta potential of (a) NLCs1 (b) NLCs9

The first generation lipid nanoparticles, solid lipid nanoparticles, had limited drug loading capacity and drug explosion during storage and these led to the emergence of second-generation nanoparticles, nanostructured lipid carriers (NLCs) [32]. NLCs have several advantages over nanoemulsions, liposomes, etc., as they show high drug loading capacity, prevents drug explosion during storage, more flexibility, and possible usage for oral delivery [32]. In this context, atorvastatin loaded NLCs [32], amphotericin B [33], triamcinolone acetonide [34], nisoldipine [35], carbamazepine [36], agomelatine [37], etc., have been developed and showed successful drug delivery. Considering these, in the present study, we developed rivastigmine loaded novel NLCs using appropriate concentrations of fish and flaxseed oil by melting emulsification coupled with high shear homogenization technique for better and prolonged drug delivery. The formation of NLCs was confirmed by phase-contrast microscopy. Particle size, SEM and TEM study confirms nano-sized discrete rod, spherical particles with smooth surface areas. FTIR spectroscopic analysis revealed the presence of functional groups in RT-NLCs. The amorphous nature of the RT-NLCs was found by DSC. Particle size analysis and zeta potential showed the average size of the RT-NLCs was 1 to $100 \mathrm{~nm}$ and-16.4 mV which indicates the stability of RT-NLCs. All our results indicate the possible and potent use of NLCs for drug delivery, especially for challenging targets such as neurons and could be used to treat AD.

\section{CONCLUSION}

All the experimental evidence indicated the possible usage of rivastigmine loaded nanostructured lipid carriers (RT-NLCs) for targeted and controlled/sustained release of drug to the brain, which would offer several advantages including an increase in drug bioavailability, drug retention capacity at the target site, improving the adherence capacity to the designated target and sustaining drug release depots.

\section{ACKNOWLEDGEMENT}

The authors are highly grateful to DST-CURIE Center, Sri Padmavati Mahila Visvavidyalayam, Tirupati for providing equipment to carry out this research work.

\section{FUNDING}

Nil

\section{AUTHORS CONTRIBUTIONS}

All the authors have contributed equally.

\section{CONFLICTS OF INTERESTS}

Declared none

\section{REFERENCES}

1. Petrasek T, Skurlova M, Maleninska K, Vojtechova I, Kristofikova Z, Matuskova H, et al. A rat model of Alzheimer's disease based on Abeta 42 and pro-oxidative substances exhibits cognitive deficit and alterations in glutamatergic and cholinergic neurotransmitter systems. Frontiers Aging Neurosci 2016;20:8-83

2. Kalaria RN, Maestre GE, Arizaga R, Friedland RP, Galasko D, Hall $\mathrm{K}$, et al. Alzheimer's disease and vascular dementia in developing countries: prevalence, management, and risk factors. Lancet Neurol 2008; 7:812-26.

3. Jack CR, Wiste HJ, Lesnick TG, Weigand SD, Knopman DS, Vemuri $\mathrm{P}$, et al. Brain $\beta$-amyloid load approaches a plateau. Neurology 2013;80:890-6.

4. Banks WA. From blood-brain barrier to blood-brain interface: new opportunities for CNS drug delivery. Nat Rev Drug Discovery 2016;15:275.

5. Pardridge WM. Alzheimer's disease drug development and the problem of the blood-brain barrier. Alzheimer's Dementia 2009;5:427-32.

6. Raschetti R, Albanese E, Vanacore N, Maggini M. Cholinesterase inhibitors in mild cognitive impairment: a systematic review of randomised trials. PLoS Med 2007;4:338.

7. Pharmaceuticals N. Inventor; Excelon monograph, USA; 2004.

8. Ismail MF, ElMeshad AN, Salem NA. Potential therapeutic effect of nano-based formulation of rivastigmine on rat model of Alzheimer's disease. Int J Nanomed 2013;8:393.

9. Arumugam K, Subramanian G, Mallayasamy S, Averineni R, Reddy M, Udupa N. A study of rivastigmine liposomes for delivery into the brain through intranasal route. Acta Pharm 2008;58:287-97.

10. Joshi SA, Chavhan SS, Sawant KK. Rivastigmine-loaded PLGA and PBCA nanoparticles: preparation, optimization, characterization, in vitro and pharmacodynamic studies. Eur J Pharm Biopharm 2010;76:189-99.

11. Sahni JK, Doggui S, Ali J, Baboota S, Dao L, Ramassamy C. Neurotherapeutic applications of nanoparticles in Alzheimer's disease. J Controlled Release 2011;152:208-31. 
12. Saraiva C, Praça C, Ferreira R, Santos T, Ferreira L, Bernardino L. Nanoparticle-mediated brain drug delivery: overcoming blood-brain barrier to treat neurodegenerative diseases. J Controlled Release 2016;235:34-47.

13. Tapeinos C, Battaglini M, Ciofani G. Advances in the design of solid lipid nanoparticles and nanostructured lipid carriers for targeting brain diseases. J Controlled Release 2017;264:306-32.

14. Velmurugan R, Selvamuthukumar S. Development and optimization of ifosfamide nanostructured lipid carriers for oral delivery using response surface methodology. Appl Nanosci 2016;6:159-73.

15. Naseri N, Valizadeh H, Zakeri Milani P. Solid lipid nanoparticles and nanostructured lipid carriers: structure, preparation and application. Adv Pharm Bull 2015;5:305

16. Fang CLA, Al-Suwayeh S, Fang JY. Nanostructured lipid carriers (NLCs) for drug delivery and targeting. Recent Patents Nanotechnol 2013;7:41-55.

17. Md S, Kit B, Jagdish S, David DJ, Pandey M, Chatterjee LA. Development and in vitro evaluation of a zerumbone loaded nanosuspension drug delivery system. Crystals 2018;8:286.

18. Chen ZY, Cao J, Xie L, Li XF, Yu ZH, Tong WY. Construction of leaky strains and extracellular production of exogenous proteins in recombinant Escherichia coli. Microbial Biotechnol 2014;7:360-70.

19. Brzobohaty O, Siler M, Trojek J, Chvatal L, Karasek V, Patak A, et al. Three-dimensional optical trapping of a plasmonic nanoparticle using low numerical aperture optical tweezers. Sci Rep 2015;29:8106.

20. Mishra P, Tyagi S. Surface analysis of gutta percha after disinfecting with sodium hypochlorite and silver nanoparticles by atomic force microscopy: an in vitro study. Dental Res J 242;15:242.

21. Kumar BS, Panda PK. 1D water chain stabilized by mesoexpanded calix [4] pyrrole. Cryst Eng Comm 2014;16:8669-72.

22. Janes KA, Fresneau MP, Marazuela A, Fabra A, Alonso MJ Chitosan nanoparticles as delivery systems for doxorubicin. J Controlled Release 2001;73:255-67.

23. Li R, Song XN, Zhang H, Dai WB, He B, Zheng Y, et al. The integrity study on PEG-PCL micelles transcellular transported across MDCK epithelial cell monolayer using FRET technology. Yao Xue Xue Bao= Acta Pharm Sinica 2016;51:1316-24.

24. Shete H, Patravale V. Long chain lipid based tamoxifen NLC. Part I: preformulation studies, formulation development and physicochemical characterization. Int J Pharm 2013;454:57383.

25. Joshi M, Pathak S, Sharma S, Patravale V. Design and in vivo pharmacodynamic evaluation of nanostructured lipid carriers for parenteral delivery of artemether: Nanoject. Int J Pharm 2008;364:119-26.
26. Subedi RK, Kang KW, Choi HK. Preparation and characterization of solid lipid nanoparticles loaded with doxorubicin. Euro J Pharm Sci 2009;37:508-13.

27. Gaba B, Fazil M, Khan S, Ali A, Baboota S, Ali J. Nanostructured lipid carrier system for topical delivery of terbinafine hydrochloride. Bull Faculty Pharm Cairo University 2015;53:147-59.

28. Bunjes $\mathrm{H}$, Unruh T. Characterization of lipid nanoparticles by differential scanning calorimetry, X-ray and neutron scattering. Adv Drug Delivery Rev 2007;59:379-402.

29. Gupta H, Aqil M, Khar RK, Ali A, Bhatnagar A, Mittal G. Sparfloxacin-loaded PLGA nanoparticles for sustained ocular drug delivery. Nanomed: Nanotechnol Biol Med 2010;6:324-33.

30. Shah NV, Seth AK, Balaraman R, Aundhia CJ, Maheshwari RA, Parmar GR. Nanostructured lipid carriers for oral bioavailability enhancement of raloxifene: design and in vivo study. J Adv Res 2016; 7:423-34.

31. Jia L, Zhang D, Li Z, Duan C, Wang Y, Feng F, et al. Nanostructured lipid carriers for parenteral delivery of silybin: Biodistribution and pharmacokinetic studie. Colloids Surf B 2010;80:213-8.

32. Elmowafy M, Ibrahim HM, Ahmed MA, Shalaby K, Salama A, Hefesha H. Atorvastatin-loaded nanostructured lipid carriers (NLCs): strategy to overcome oral delivery drawbacks. Drug Delivery 2017;24:932-41.

33. Jansook P, Pichayakorn W, Ritthidej GC. Amphotericin B-loaded solid lipid nanoparticles (SLNs) and nanostructured lipid carrier (NLCs): effect of drug loading and biopharmaceutical characterizations. Drug Dev Industrial Pharm 2018;44:1693-700.

34. Kraisit P, Sarisuta N. Development of triamcinolone acetonideloaded nanostructured lipid carriers (NLCs) for buccal drug delivery using the Box-Behnken design. Molecules 2018;23:982.

35. Dudhipala N, Janga KY, Gorre T. Comparative study of nisoldipine-loaded nanostructured lipid carriers and solid lipid nanoparticles for oral delivery: preparation, characterization, permeation and pharmacokinetic evaluation. Artificial Cells Nanomed Biotechnol 2018;46:616-25.

36. Montoto SS, Sbaraglini ML, Talevi A, Couyoupetrou M, Di Ianni M, Pesce GO, et al. Carbamazepine-loaded solid lipid nanoparticles and nanostructured lipid carriers: physicochemical characterization and in vitro/in vivo evaluation. Colloids Surf B 2018;167:73-81.

37. Prajapati JB, Verma SD, Patel AA. Oral bioavailability enhancement of agomelatine by loading into nanostructured lipid carriers: peyer's patch targeting approach. Int J Nanomed 2018;13:35-8. 